\title{
Optical Modeling for Polarization-dependent Optical Power Dissipation of Thin-film Organic Solar Cells at Oblique Incidence
}

\author{
Jungho Kim*, Sungyeop Jung, and Inkyung Jeong \\ Department of Information Display, Kyung Hee University, Seoul 130-701, Korea
}

(Received November 16, 2011 : revised January 5, 2012 : accepted January 5, 2012)

\begin{abstract}
We present the optical models and calculation results of thin-film organic solar cells (OSCs) at oblique incidence of light, using the transfer matrix method. The simple expression for the optical power dissipation is derived at oblique incidence for s- and p-polarized light. The spatial distribution of the electric field intensity, the optical power density, and the optical power dissipation are calculated in both s- and p-polarized light with respect to the incidence angle. We identify how the light absorption efficiency for p-polarized light becomes relatively larger than that for s-polarized light as the incidence angle increases.
\end{abstract}

Keywords: Organic solar cell, Optical modeling, Oblique incidence, Transfer matrix method OCIS codes : (040.5350) Photovoltaic; (200.4860) Optical vector-matrix systems; (310.6805) Theory and design

\section{INTRODUCTION}

Organic solar cells (OSCs) have been intensively investigated as one of the promising green energy sources due to the feasibility of producing thin, light-weight, and flexible solar cells [1]. However, the power conversion efficiency should be further improved for practical use of the OSC. Optical interference effects play an important role in the efficiency of multilayer OSCs, where the thickness of each thin-film layer should be carefully designed to maximize the optical absorption in the active region.

The optical interference effect of the multilayer solar cells has been modeled based on the finite element method (FEM) [2, 3], the rigorous coupled-wave analysis (RCWA) $[4,5]$, and the transfer matrix method (TMM) [6-8]. Among them, the TMM has been widely used for thin-film OSCs due to its relatively ease of simulation code implementation but high accuracy in calculation results. The TMM-based optical model for the OSC provides the spatial distribution of the time-average optical power dissipation $Q(z)$ usually for normal incidence of sunlight [6-8]. Here, $Q(z)$ is related with the amount of photon energy absorbed by the material per unit time and per unit volume and $z$ is a position within the multilayer OSC.

Recently, the angular response of thin-film OSCs with respect to the incidence angle of sunlight has been intensively investigated due to the fact that most OSCs, having the advantage of low-cost production and flexible application, are expected to be used without a sunlight tracking system. The TMM-based optical model for normal incidence has been adopted for oblique incidence to analyze the angular response of thin-film OSCs [9-11]. However, the previous TMM-based optical models for oblique incidence of sunlight are still insufficient.

It was mathematically proved that $Q(z)$ can be expressed as $Q(z)=(1 / 2) c \varepsilon_{0} n \alpha|E(z)|^{2}$ for normal incidence of sunlight [6]. Here, $c$ is the speed of light in free space, $\varepsilon_{0}$ is the permittivity in free space, $n$ is the real part of the complex refractive index, $\alpha$ is the absorption coefficient, and $E(z)$ is the electric field amplitude at the position $z$. In the case of oblique incidence, $Q(z)$ was also assumed to be proportional to $|E(z)|^{2}$ for both s- and p-polarized sunlight $[10,11]$. However, we have not yet found any text to provide a clear derivation for the relation between $Q(z)$ and $|E(z)|^{2}$ at oblique incidence, especially for p-polarized light. In addition, the effect of the incidence angle and light polarization on the distribution of $Q(z)$ has not yet been investigated quantitatively.

In this paper, we present comprehensive optical modeling and calculation results of thin-film OSCs at oblique incidence

\footnotetext{
*Corresponding author: junghokim@khu.ac.kr

Color versions of one or more of the figures in this paper are available online.
} 
of sunlight. By applying the TMM at oblique incidence, we derive a simple expression for $Q(z)$ for both s- and p-polarized light. We calculate the spatial distribution of the electric field intensity, the time-average Poynting vector (optical power density), and the optical power dissipation with respect to the incidence angle. We investigate how the optical interference effect of thin-film OSCs is affected by the incidence angle and light polarization.

\section{OPTICAL MODEL}

Fig. 1 shows a stack of $M$ layers, where a plane wave with an incident angle $\theta$ and a wavelength $\lambda$ is incident from the semi-infinite transparent ambient on the left $(\mathrm{j}=0)$. The multilayer stack is assumed to be isotropic and homogeneous with plane and parallel interfaces. The orientation of the electromagnetic field oscillations is composed of s-polarization and p-polarization that are perpendicular to or parallel to the incident plane, respectively. Each thin-film layer $\mathrm{j}(\mathrm{j}=1,2, \cdots, \mathrm{M})$ has a thickness of $d_{j}$ and a complex refractive index of $\tilde{n}_{j}=n_{j}+i \kappa_{j}$, where $n_{j}$ and $\kappa_{j}$ are the refractive index and extinction coefficient, respectively.

In Fig. 1, the wave vector in the transparent ambient of the 0 -th layer is given by

$$
\begin{aligned}
& \vec{k}_{0}=\left(k_{x, 0}, 0, k_{z, 0}\right), \\
& k_{x, 0}=n_{0} \sin \theta k_{0}, \\
& k_{z, 0}=n_{0} \cos \theta k_{0},
\end{aligned}
$$

where $n_{0}$ is the refractive index of the 0 -th layer and $k_{0}=2 \pi / \lambda$. In the $\mathrm{j}$-th layer, the wave vector is written as

$$
\begin{aligned}
& \vec{k}_{j}=\left(k_{x, j}, 0, k_{z, j}\right), \\
& k_{x, j}=k_{x, 0}=n_{0} \sin \theta k_{0}, \\
& k_{z, j}=\tilde{n}_{j} \cos \theta_{j} k_{0}=\left(p_{j}+i q_{j}\right) k_{0},
\end{aligned}
$$

where the relation $k_{x, j}=k_{x, 0}$ is ascribed to the boundary condition that the transverse wave vector should be continuous at the interface. The terms $p_{j}=\operatorname{Re}\left\{\tilde{n}_{j} \cos \theta_{j}\right\}$ and $q_{j}=\operatorname{Im}\left\{\tilde{n}_{j} \cos \theta_{j}\right\}$ correspond to the effective refractive index and the extinction coefficient for the $\mathrm{z}$ direction. From the dispersion relation $k_{x, j}^{2}+k_{z, j}^{2}=\tilde{n}_{j}^{2} k_{0}^{2}$, we have $p_{j}+i q_{j}=\sqrt{\left(n_{j}+i \kappa_{j}\right)^{2}-\left(n_{0} \sin \theta\right)^{2}}$. Consequently, we obtain

$$
\begin{aligned}
& p_{j}^{2}-q_{j}^{2}=n_{j}^{2}-\kappa_{j}^{2}-\left(n_{0} \sin \theta\right)^{2}, \\
& p_{j} q_{j}=n_{j} \kappa_{j} .
\end{aligned}
$$

\subsection{Internal Reflection and Transmission Coefficients in Layer $\mathbf{j}$}

In Fig. 1, we assign a positive (negative) direction to the light propagating from left (right) to right (left), designating the $+(-)$ superscripts. The electric field amplitudes on the interface between the adjacent layer $\mathrm{j}$ and $\mathrm{k}=\mathrm{j}+1$ are described by an interface matrix $\mathbf{I}^{\mathrm{jk}}$ :

$$
\left[\begin{array}{c}
E_{j R}^{+} \\
E_{j R}^{-}
\end{array}\right]=\mathbf{I}^{j k}\left[\begin{array}{c}
E_{k L}^{+} \\
E_{k L}^{-}
\end{array}\right]=\frac{1}{t_{j k}}\left[\begin{array}{cc}
1 & r_{j k} \\
r_{j k} & 1
\end{array}\right]\left[\begin{array}{c}
E_{k L}^{+} \\
E_{k L}^{-}
\end{array}\right],
$$

where $E_{j R}^{+}$and $E_{k L}^{+}$are the forward-propagating electric field amplitudes at the right boundary of the layer $\mathrm{j}$ and the left boundary of the layer $\mathrm{k}$, respectively. The terms $r_{j k}$

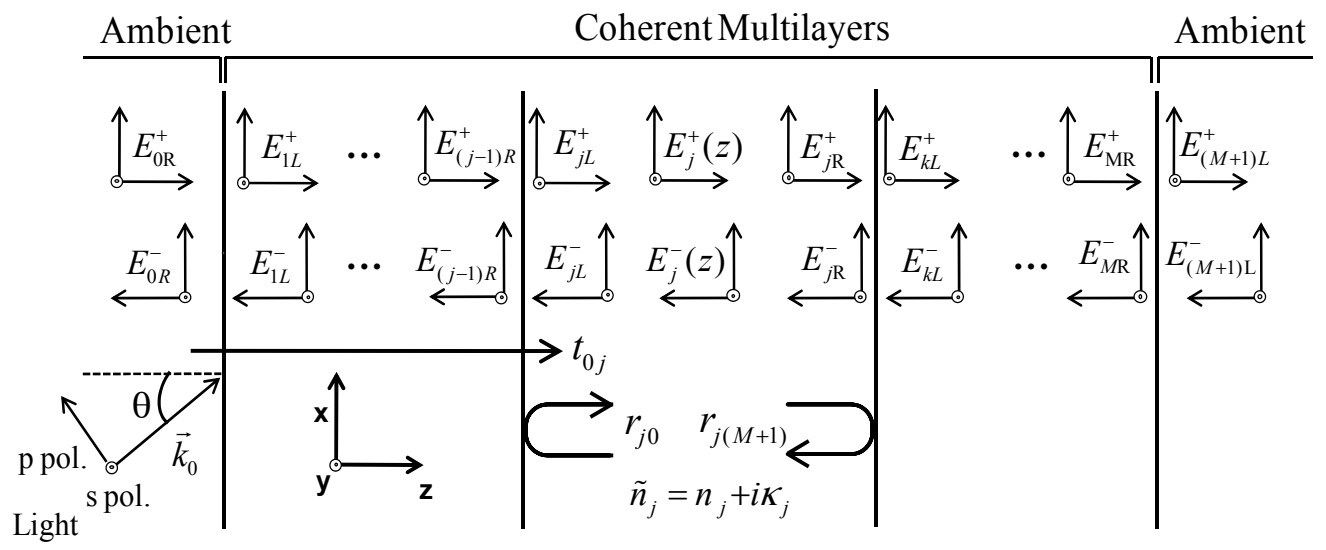

FIG. 1. Schematic diagram of M multilayers with forward- and backward-propagating electric field amplitude $(E)$. A plane wave with an incident angle of $\theta$ and a wavelength of $\lambda$ is incident from the semi-infinite transparent ambient on the left $(\mathrm{j}=0)$. The orientation of the electromagnetic field oscillations is composed of s-polarization and p-polarization that are perpendicular to or parallel to the incident plane, respectively. 
and $t_{j k}$ are the complex Fresnel reflection and transmission coefficients on the interface of the adjacent layer. For s-polarized light, they are defined as [12]

$$
\begin{aligned}
r_{j k}^{s} & =\frac{\tilde{n}_{j} \cos \theta_{j}-\tilde{n}_{k} \cos \theta_{k}}{\tilde{n}_{j} \cos \theta_{j}+\tilde{n}_{k} \cos \theta_{k}}, \\
t_{j k}^{s} & =\frac{2 \tilde{n}_{j} \cos \theta_{j}}{\tilde{n}_{j} \cos \theta_{j}+\tilde{n}_{k} \cos \theta_{k}}
\end{aligned}
$$

and for p-polarized light as

$$
\begin{aligned}
& r_{j k}^{p}=\frac{\tilde{n}_{j} \cos \theta_{k}-\tilde{n}_{k} \cos \theta_{j}}{\tilde{n}_{j} \cos \theta_{k}+\tilde{n}_{k} \cos \theta_{j}}, \\
& t_{j k}^{p}=\frac{2 \tilde{n}_{j} \cos \theta_{j}}{\tilde{n}_{j} \cos \theta_{k}+\tilde{n}_{k} \cos \theta_{j}} .
\end{aligned}
$$

The propagation of the electric field amplitude between the left and right boundaries of the $\mathrm{j}$-th layer is described by a layer matrix $\mathbf{L}^{\mathrm{j}}$ :

$$
\left[\begin{array}{c}
E_{j R}^{+} \\
E_{j R}^{-}
\end{array}\right]=\mathbf{L}^{j}\left(d_{j}\right)\left[\begin{array}{c}
E_{j L}^{+} \\
E_{j L}^{-}
\end{array}\right]=\left[\begin{array}{cc}
e^{-i k_{z, j} d_{j}} & 0 \\
0 & e^{i k_{z, j} d_{j}}
\end{array}\right]\left[\begin{array}{c}
E_{j L}^{+} \\
E_{j L}^{-}
\end{array}\right],
$$

where we assume the time dependence of $e^{i w t}$.

In Fig. 1, the electric field amplitude passing through all the $\mathrm{M}$ multilayers from the ambient on the left (layer 0 ) to the right (layer $\mathrm{M}+1$ ) can be written as

$$
\left[\begin{array}{c}
E_{0 R}^{+} \\
E_{0 R}^{-}
\end{array}\right]=\mathbf{S}^{0 /(M+1)}\left[\begin{array}{l}
E_{(M+1) L}^{+} \\
E_{(M+1) L}^{-}
\end{array}\right]
$$

where the amplitude scattering matrix $\mathbf{S}^{\mathbf{0}(M+1)}$ is defined as

$$
\mathbf{S}^{0 /(M+1)}=\left[\begin{array}{ll}
S_{11}^{0 /(M+1)} & S_{12}^{0 /(M+1)} \\
S_{21}^{0 /(M+1)} & S_{22}^{0 /(M+1)}
\end{array}\right]=\mathbf{I}^{01} \mathbf{L}^{1} \mathbf{I}^{12} \cdots \mathbf{L}^{M} \mathbf{I}^{M(M+1)} .
$$

If there is no incidence of light from the ambient on the right (layer $\mathrm{M}+1$ ) to the left (layer 0 ), $E_{(M+1) L}^{-}=0$ and the front-reflection and front-transmission coefficients from the layer 0 to $\mathrm{M}+1$ can be obtained by

$$
\begin{aligned}
& r_{0(M+1)}=\frac{E_{0 R}^{-}}{E_{0 R}^{+}}=\frac{S_{21}^{0 /(M+1)}}{S_{11}^{0 /(M+1)}}, \\
& \mathrm{t}_{0(M+1)}=\frac{E_{(M+1) L}^{+}}{E_{0 R}^{+}}=\frac{1}{S_{11}^{0 /(M+1)}} .
\end{aligned}
$$

In the same manner, we can define the complex reflection and transmission coefficients for the $\mathrm{j}$-th layer in terms of the matrix elements

$$
\begin{aligned}
& \mathrm{t}_{0 j}=\frac{1}{S_{11}^{0 / j}}, \\
& \mathbf{S}^{0 / j}=\left[\begin{array}{ll}
S_{11}^{0 / j} & S_{12}^{0 / j} \\
S_{21}^{0 / j} & S_{22}^{0 / j}
\end{array}\right]=\mathbf{I}^{01} \mathbf{L}^{1} \mathbf{I}^{12} \cdots \mathbf{L}^{(j-1)} \mathbf{I}^{(j-1) j}, \\
& \mathrm{r}_{j(M+1)}=\frac{S_{21}^{j /(M+1)}}{S_{11}^{j /(M+1)}}, \\
& \mathbf{S}^{j /(M+1)}=\left[\begin{array}{ll}
S_{11}^{j /(M+1)} & S_{12}^{j /(M+1)} \\
S_{21}^{j /(M+1)} & S_{22}^{j /(M+1)}
\end{array}\right]=\mathbf{I}^{(j+1)} \mathbf{L}^{(j+1)} \mathbf{I}^{(j+1)(j+2)} \cdots \mathbf{L}^{M} \mathbf{I}^{M(M+1)}, \\
& \mathbf{r}_{j 0}=-\mathrm{r}_{0 j}=-\frac{S_{21}^{0 / j}}{S_{11}^{0 / j}}, \\
& \mathbf{S}^{0 / j}=\left[\begin{array}{ll}
S_{11}^{0 / j} & S_{12}^{0 / j} \\
S_{21}^{0 / j} & S_{22}^{0 / j}
\end{array}\right]=\mathbf{I}^{01} \mathbf{L}^{1} \mathbf{I}^{12} \cdots \mathbf{L}^{(j-1)} \mathbf{I}^{(j-1) j} .
\end{aligned}
$$

As shown in Fig. 1, $t_{0 j}$ is the internal transmission coefficient from the ambient on the left (layer 0 ) to layer $\mathrm{j}$. The terms $r_{j 0}$ and $r_{j(M+1)}$ are the internal reflection coefficients through the partial front multilayers from layer $\mathrm{j}$ to layer 0 and through the partial rear multilayers from layer $\mathrm{j}$ to layer $\mathrm{M}+1$, respectively.

\subsection{Optical Power Dissipation for S-polarized Light}

The y component of the electric field in the $\mathrm{j}$-th layer is expressed as

$$
\begin{aligned}
E_{y, j} & =\left[t_{j}^{+} e^{i k_{z, j} z}+t_{j}^{-} e^{-i k_{z, j} z}\right] e^{i k_{x, 0} x} \\
& =\left[t_{j}^{+} e^{i p_{j} k_{0} z} e^{-q_{j} k_{0} z}+t_{j}^{-} e^{-i p_{j} k_{0} z} e^{+q_{j} k_{0} z}\right] e^{i k_{x, 0} x},
\end{aligned}
$$

where $t_{j}^{+(-)}$is an internal transfer coefficient, relating the incident plane wave to the internal electric field propagating in the positive (negative) $\mathrm{z}$ direction at the interface between the layer $\mathrm{j}-1$ and $\mathrm{j}$ [13]. The values of $t_{j}^{+}$and $t_{j}^{-}$are given by

$$
\begin{aligned}
t_{j}^{+} & =\frac{t_{0 j}}{1-r_{j 0} r_{j(M+1)} e^{2 i k_{z, j} d_{j}}}, \\
t_{j}^{-} & =t_{j}^{+} r_{j(M+1)} e^{2 i k_{z, j} d_{j}},
\end{aligned}
$$

where $t_{0 j}, r_{j(M+1)}$, and $r_{j 0}$ are defined in Eqs. (11). The corresponding magnetic fields are

$$
\begin{aligned}
H_{x, j} & =\frac{1}{i \omega \mu_{0}}\left(\frac{\partial E_{z}}{\partial y}-\frac{\partial E_{y}}{\partial z}\right) \\
& =-\frac{k_{z, j}}{\omega \mu_{0}}\left[t_{j}^{+} e^{i k_{z, j} z}-t_{j}^{-} e^{-i k_{z, j} z}\right] e^{i k_{x, 0} x} \\
& =-c \varepsilon_{0}\left(p_{j}+i q_{j}\right)\left[t_{j}^{+} e^{i p_{j} k_{0} z} e^{-q_{j} k_{0} z}-t_{j}^{-} e^{-i p_{j} k_{0} z} e^{+q_{j} k_{0} z}\right] e^{i k_{x, 0} x},
\end{aligned}
$$




$$
\begin{aligned}
H_{z, j} & =\frac{1}{i \omega \mu_{0}}\left(\frac{\partial E_{y}}{\partial x}-\frac{\partial E_{x}}{\partial y}\right) \\
& =\frac{k_{x, 0}}{\omega \mu_{0}}\left[t_{j}^{+} e^{i k_{z, j} z}+t_{j}^{-} e^{-i k_{z, j} z}\right] e^{i k_{x, 0} x} \\
& =c \varepsilon_{0} n_{0} \sin \theta\left[t_{j}^{+} e^{i p_{j} k_{0} z} e^{-q_{j} k_{0} z}+t_{j}^{-} e^{-i p_{j} k_{0} z} e^{+q_{j} k_{0} z}\right] e^{i k_{x, 0} x}
\end{aligned}
$$

The time-average Poynting vector in the $\mathrm{z}$ direction is expressed as

$$
\begin{aligned}
S_{z, j}^{s}(z) & =-\frac{1}{2} \operatorname{Re}\left\{E_{y, j} H_{x, j}^{*}\right\} \\
& =\frac{1}{2} c \varepsilon_{0} \operatorname{Re}\left\{\left(p_{j}-i q_{j}\right)\left[\left|t_{j}^{+}\right|^{2} e^{-2 q k_{j} z}-\left|t_{j}^{-}\right|^{2} e^{+2 q_{j} k_{0} z}-t_{j}^{+} t_{j}^{-*} e^{i 2 p_{j} k_{0} z}+t_{j}^{t_{j}^{*}} t_{j}^{-} e^{-i 2 p_{j} k_{0} z}\right]\right\} \\
& \left.=\frac{1}{2} c \varepsilon_{0} p_{j}\left[\left|t_{j}^{+}\right|^{2} e^{-2 q k_{j} z}-\left|t_{j}^{-}\right|^{2} e^{+2 q_{j} k_{0} z}\right]-\frac{1}{2} c \varepsilon_{0} 2 q_{j}\left|\rho_{j}\right| \sin \left(2 p_{j} k_{0} z+\delta_{j}\right)\right],
\end{aligned}
$$

where we can define $t_{j}^{+} t_{j}^{* *}=\left|\rho_{j}\right| e^{i \delta j}$. Finally, the optical power dissipation in the $\mathrm{z}$ direction is given by

$$
\begin{aligned}
Q_{z, j}^{s}(z) & =-\frac{d S_{z, j}^{s}}{d z} \\
& =\frac{1}{2} c \varepsilon_{0} p_{j}\left(2 q_{j} k_{0}\right)\left[\left|t_{j}^{+}\right|^{2} e^{-2 q q_{k_{0}} z}+\left|t_{j}^{-}\right|^{2} e^{2 q, k_{0,0} z}\right]+\frac{1}{2} c \varepsilon_{0} p_{j}\left(2 q_{j} k_{0}\right) 2\left|\rho_{j}\right| \cos \left(2 p_{j} k_{0} z+\delta_{j}\right) \\
& =\frac{1}{2} c \varepsilon_{0} p_{j} \alpha_{j, \theta}\left[\left|t_{j}^{+}\right|^{2} e^{-2 q_{j} k_{0} z}+\left|t_{j}^{-}\right|^{2} e^{2 q_{j} k_{0} z}+2\left|\rho_{j}\right| \cos \left(2 p_{j} k_{0} z+\delta_{j}\right)\right] \\
& =\frac{1}{2} c \varepsilon_{0} p_{j} \alpha_{j, \theta}\left|E_{y, j}\right|^{2}
\end{aligned}
$$

where $p_{j}=\operatorname{Re}\left\{\tilde{n}_{j} \cos \theta_{j}\right\}$ and $\alpha_{j, \theta}=2 q_{\mathrm{j}} k_{0}$ in Eq. (18) are the real part of the complex refractive index and the absorption coefficient at the incident angle $\theta$, respectively. We can obtain Eq. (19) by substituting Eq. (12) into Eq. (18). Similar to normal incidence, the optical power dissipation at the incident angle $\theta$ is also proportional to the electric field intensity for s-polarized light. It is noticeable that the values of $p_{j}$ and $\alpha_{j, \theta}$ are modified for oblique incidence compared with the case of normal incidence.

\subsection{Optical Power Dissipation for P-polarized Light}

The $\mathrm{x}$ and $\mathrm{z}$ component of the magnetic field in the $\mathrm{j}$-th layer can be written as

$$
\begin{aligned}
E_{x, j} & =\frac{k_{z, j}}{\left(n_{j}+i \kappa_{j}\right) k_{0}}\left[t_{j}^{+} e^{i k_{z, j} z}+t_{j}^{-} e^{-i k_{z, j} z}\right] e^{i k_{x, 0} x} \\
& =\frac{p_{j}+i q_{j}}{\left(n_{j}+i \kappa_{j}\right)}\left[t_{j}^{+} e^{i p_{j} k_{0} z} e^{-q_{j} k_{0} z}+t_{j}^{-} e^{-i p_{j} k_{0} z} e^{+q_{j} k_{0} z}\right] e^{i k_{x, 0} x}, \\
E_{z, j} & =\frac{k_{k, 0}}{\left(n_{j}+i \kappa_{j}\right) k_{0}}\left[-t_{j}^{+} e^{i k_{z, j} z}+t_{j}^{-} e^{-i k_{z, j} z}\right] e^{i k_{x, 0} x} \\
& =\frac{n_{0} \sin \theta}{\left(n_{j}+i \kappa_{j}\right)}\left[-t_{j}^{+} e^{i p_{j} k_{0} z} e^{-q_{j} k_{0} z}+t_{j}^{-} e^{-i p_{j} k_{0} z} e^{+q_{j} k_{0} z}\right] e^{i k_{x, 0} x} .
\end{aligned}
$$

The corresponding magnetic field is given by

$$
\begin{aligned}
H_{x, j} & =\frac{1}{i \omega \mu_{0}}\left(\frac{\partial E_{x}}{\partial z}-\frac{\partial E_{z}}{\partial x}\right) \\
& =\frac{k_{z, j}^{2}+k_{x, 0}^{2}}{\omega \mu_{0}\left(n_{j}+i \kappa_{j}\right) k_{0}}\left[t_{j}^{+} e^{i k_{z, j} z}-t_{j}^{-} e^{-i k_{z, j} z}\right] e^{i k_{x, 0} x} \\
& =\frac{\tilde{n}_{j}^{2} k_{0}^{2}}{\omega \mu_{0}\left(n_{j}+i \kappa_{j}\right) k_{0}}\left[t_{j}^{+} e^{i k_{z, j} z}-t_{j}^{-} e^{-i k_{z, j} z}\right] e^{i k_{x, 0} x} \\
& =\frac{\left(n_{j}+i \kappa_{j}\right)^{2} k_{0}^{2}}{\omega \mu_{0}\left(n_{j}+i \kappa_{j}\right) k_{0}}\left[t_{j}^{+} e^{i k_{z, j} z}-t_{j}^{-} e^{-i k_{z, j} z}\right] e^{i k_{x, 0} x} \\
& =c \varepsilon_{0}\left(n_{j}+i \kappa_{j}\right)\left[t_{j}^{+} e^{i p_{j} k_{0} z} e^{-q_{j} k_{0} z}-t_{j}^{-} e^{-i p_{j} k_{0} z} e^{+q_{j} k_{0} z}\right] e^{i k_{x, 0} x} .
\end{aligned}
$$

The time-average Poynting vector in the $\mathrm{z}$ direction is given by

$$
\begin{aligned}
& S_{z, j}^{p}(z)=\frac{1}{2} \operatorname{Re}\left\{E_{x, j} H_{y, j}^{*}\right\} \\
& =\frac{1}{2} c \varepsilon_{0} \operatorname{Re}\left\{\frac{\left(p_{j}+i q_{j}\right)}{\left(\eta_{j}+i \kappa_{j}\right)}\left(n_{j}-i \kappa_{j}\right)\left[\left|t_{j}^{+}\right|^{2} e^{-2 q, k_{0} z}-\left|t_{j}^{-}\right|^{2} e^{+2 q q k_{0} z}-t_{j}^{+} t_{j}^{-*} e^{i 2 p, k_{0} z}+t_{j}^{+*} t_{j}^{-} e^{-i 2 p, k_{j} z}\right]\right\} \\
& =\frac{1}{2} c \varepsilon_{0} \operatorname{Re}\left\{\frac{\left(p_{j}+i q_{j}\right)}{\left(\eta_{j}+i \kappa_{j}\right)}\left(n_{j}-i \kappa_{j}\right)\right\}\left[\left|t_{j}^{+}\right|^{2} e^{-2 q_{j} k_{0} z_{0}}-\left|t_{j}^{-}\right|^{2} e^{+2 q_{j} k_{0} \alpha^{2}}\right] \\
& +\frac{1}{2} c \varepsilon_{0} \operatorname{Im}\left\{\frac{\left(p_{j}+i q_{j}\right)}{\left(n_{j}+i K_{j}\right)}\left(n_{j}-i \kappa_{j}\right)\right\} 2\left|\rho_{j}\right| \sin \left(2 p_{j} k_{0} z+\delta_{j}\right) \text {. }
\end{aligned}
$$

Finally, the optical power dissipation in the $\mathrm{z}$ direction is written as

$$
\begin{aligned}
Q_{z, j}^{p}(z)= & -\frac{d S_{z, j}^{p}}{d z} \\
= & \frac{1}{2} c \varepsilon_{0}\left(2 q_{j} k_{0}\right) \operatorname{Re}\left\{\frac{\left(p_{j}+i q_{j}\right)}{\left(n_{j}+i \kappa_{j}\right)}\left(n_{j}-i \kappa_{j}\right)\right\}\left[\left|t_{j}^{+}\right|^{2} e^{-2 q_{j} k_{0} z}+\left|t_{j}^{-}\right|^{2} e^{+2 q_{j} k_{0} z}\right] \\
& -\frac{1}{2} c \varepsilon_{0}\left(2 p_{j} k_{0}\right) \operatorname{Im}\left\{\frac{\left(p_{j}+i q_{j}\right)}{\left(n_{j}+i \kappa_{j}\right)}\left(n_{j}-i \kappa_{j}\right)\right\} 2\left|\rho_{j}\right| \cos \left(2 p_{j} k_{0} z+\delta_{j}\right) \\
= & \frac{1}{2} c \varepsilon_{0}\left(2 q_{j} k_{0}\right) \frac{2 q_{j} n_{j} \kappa_{j}+p_{j}\left(n_{j}^{2}-\kappa_{j}^{2}\right)}{n_{j}^{2}+\kappa_{j}^{2}}\left[\left|t_{j}^{+}\right|^{2} e^{-2 q_{j} k_{0} z}+\left|t_{j}^{-}\right|^{2} e^{+2 q_{j} k_{0} z}\right] \\
& +\frac{1}{2} c \varepsilon_{0} \frac{2 p_{j} n_{j} \kappa_{j}-q_{j}\left(n_{j}^{2}-\kappa_{j}^{2}\right)}{n_{j}^{2}+\kappa_{j}^{2}}\left(2 p_{j} k_{0}\right) 2\left|\rho_{j}\right| \cos \left(2 p_{j} k_{0} z+\delta_{j}\right) .
\end{aligned}
$$

Rearrangement of Eq. (24) together with Eq. (3) leads to

$$
\begin{aligned}
Q_{z, j}^{p}(z)= & \frac{1}{2} c \varepsilon_{0} p_{j}\left(2 q_{j} k_{0}\right) \frac{p_{j}^{2}+q_{j}^{2}+\left(n_{0} \sin \theta\right)^{2}}{n_{j}^{2}+\kappa_{j}^{2}}\left[\left|t_{j}^{+}\right|^{2} e^{-2 q q_{0} k_{0} z}+\left|t_{j}\right|^{2} e^{+2 q q_{j} k_{0} z}\right] \\
& +\frac{1}{2} c \varepsilon_{0} p_{j}\left(2 q_{j} k_{0}\right) \frac{p_{j}^{2}+q_{j}^{2}-\left(n_{0} \sin \theta\right)^{2}}{n_{j}^{2}+\kappa_{j}^{2}} 2\left|\rho_{j}\right|^{2} \cos \left(2 p_{j} k_{0} z+\delta_{j}\right) \\
= & \frac{1}{2} c \varepsilon_{0} p_{j}\left(2 q_{j} k_{0}\right) \frac{p_{j}^{2}+q_{j}^{2}}{n_{j}^{2}+\kappa_{j}^{2}}\left[\left|t_{j}^{+}\right|^{2} e^{-2 q k_{0} z}+\left|t_{j}\right|^{2} e^{+2 q, k_{0} z}+2\left|\rho_{j}\right| \cos \left(2 p_{j} k_{0} z+\delta_{j}\right)\right] \\
& +\frac{1}{2} c \varepsilon_{0} p_{j}\left(2 q_{j} k_{0}\right) \frac{\left(n_{0} \sin \theta\right)^{2}}{n_{j}^{2}+\kappa_{j}^{2}}\left[\left|t_{j}^{+}\right|^{2} e^{-2 q k_{j} k_{0} z}+\left|t_{j}^{-}\right|^{2} e^{+2 q k_{j} z}-2\left|\rho_{j}\right| \cos \left(2 p_{j} k_{0} z+\delta_{j}\right)\right] .
\end{aligned}
$$

By substituting the expressions for $E_{x, j}$ and $E_{z, j}$ in Eqs. (20) and (21) into Eq. (25), we obtain 


$$
\begin{aligned}
& Q_{z, j}^{p}(z)=\frac{1}{2} c \varepsilon_{0} p_{j}\left(2 q_{j} k_{0}\right)\left|\frac{\left(p_{j}+i q_{j}\right)}{\left(n_{j}+i \kappa_{j}\right)}\left[t_{j}^{+} e^{i p, k_{0} z} e^{-q, k_{1} z}+t_{j}^{-} e^{-i p, k_{0} z} e^{+q, k_{0} z}\right] e^{i k_{x, x},\left.\right|^{2}}\right|^{2} \\
& +\frac{1}{2} c \varepsilon_{0} p_{j}\left(2 q_{j} k_{0}\right)\left|\frac{n_{0} \sin \theta}{\left(n_{j}+i K_{j}\right)}\left[-t_{j}^{+} e^{i p_{j} k_{0} z} e^{-q, k_{k} k_{0} z}+t_{j}^{-} e^{-i p, k_{0} z} e^{+q, k_{0} x_{0}}\right] e^{i k_{k, 0} x}\right|^{2} \\
& =\frac{1}{2} c \varepsilon_{0} p_{j} \alpha_{j, \theta}\left(\left|E_{x, j}\right|^{2}+\left|E_{z, j}\right|^{2}\right) \text {. }
\end{aligned}
$$

It is remarkable that the optical power dissipation for p-polarized light at oblique incidence is also proportional to the electric field intensity, which is composed of the $x$ and $\mathrm{z}$ components of the electric field amplitude for p-polarized light. Thus, information on the electric field intensity with respect to light polarization and incident angle is sufficient to calculate the optical power dissipation in the OSC.

\subsection{Total Light Absorptance at the Active Region}

Because light absorbed in the active region of the OSC can contribute to the electric power generation, we have to maximize the light absorption efficiency at the active region to enhance power conversion efficiency of the OSC. The light absorptance of the layer $\mathrm{j}\left(\mathrm{d}_{\mathrm{j}-1} \leq{ }_{z} \leq d_{\mathrm{j}}\right)$ can be expressed as [8]

$$
A_{j}=\frac{1}{S_{0}^{s(p)}} \int_{d_{j-1}}^{d_{j}} Q_{j}^{s(p)}(z) d z
$$

where $S_{0}^{s(p)}$ is the optical power of sunlight light with $\mathrm{s}(\mathrm{p})$ polarization, which is incident from the ambient on the left.

\section{CALCULATION RESULTS}

Fig. 2 shows the multilayer structure of the OSC along with its corresponding complex refractive index, which is

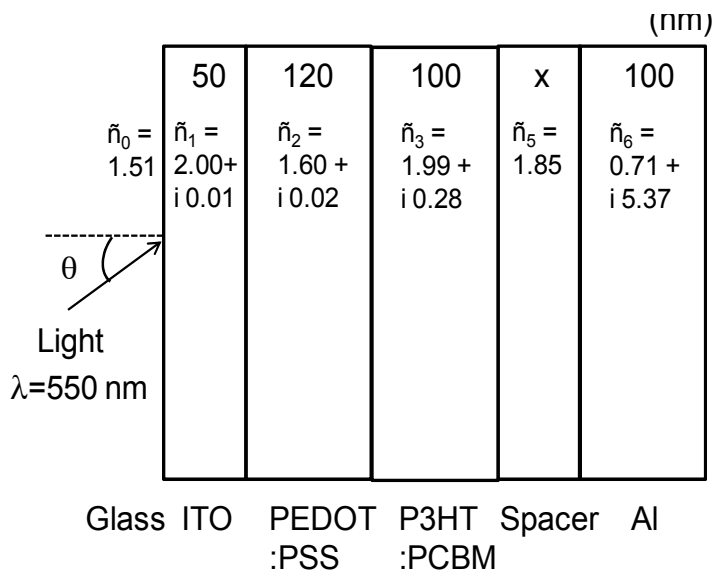

FIG. 2. Multilayer structure of the OSC together with its corresponding complex refractive index. The thickness of the optical spacer layer is varied as $\mathrm{x}=50,100$, and $150 \mathrm{~nm}$. taken from Ref. 14. The wavelength of light is assumed to be $550 \mathrm{~nm}$, where the P3HT:PCBM active layer has a peak absorption. We consider the incident angles of $0^{\circ}, 30^{\circ}, 45^{\circ}$, and $60^{\circ}$ in this calculation. The thickness of the optical spacer layer is varied as $\mathrm{x}=50,100$, and $150 \mathrm{~nm}$ to adjust the optical interference effect and maximize the electric field intensity distribution within the active region.

Fig. 3 shows the y component of the normalized electric field intensities at the spacer thickness of 50, 100, and 150 $\mathrm{nm}$. The light green area in the middle of Fig. 3 indicates the P3HT:PCBM active region, where the electric field intensity distribution is important for determining the efficiency of the OSC. The value of the electric field intensity is normalized to s-polarized light incident from the transparent glass on the left ambient when the incident angle is (a) $0^{\circ}$, (b) $30^{\circ}$ and (c) $60^{\circ}$. From the ITO toward the Al layers, the oscillating electric field intensities show the general interference pattern [6-8]. For normal incidence $\left(\theta=0^{\circ}\right)$, the spatial distribution of $\left|E_{y}\right|^{2}$ in the P3HT:PCBM active region is the largest at the spacer thickness of $100 \mathrm{~nm}$, where the OSC expects to have the best light absorption efficiency. As the incident angle increases to $30^{\circ}$ and further up to $60^{\circ}$, the overall oscillating interference pattern of $\left|E_{y}\right|^{2}$ moves toward the ITO layer. This behavior is ascribed to the fact that the real part of the complex refractive index, $\mathrm{p}_{\mathrm{j}}=\mathrm{Re}$ $\left\{\tilde{n}_{j} \cos \theta_{j}\right\}$, becomes smaller at the larger incident angle.

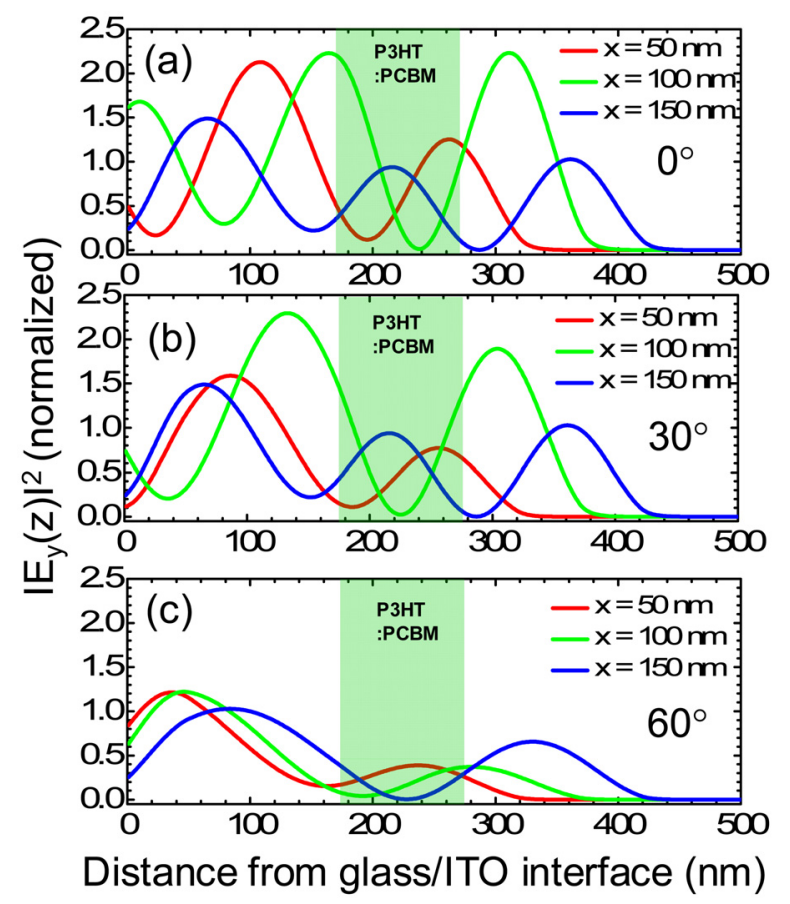

FIG. 3. The y component of the normalized electric field intensities at the spacer thicknesses of 50,100, and $150 \mathrm{~nm}$. The light green area indicates the P3HT:PCBM active region. The value of the electric field intensity is normalized to s-polarized light incident from the transparent glass at the incident angles of (a) $0^{\circ}$, (b) $30^{\circ}$ and (c) $60^{\circ}$. 
Fig. 4 shows the time-average Poynting vectors $S_{z}(z)$ for s- and p-polarized light at the incident angles of $0^{\circ}, 30^{\circ}$, $45^{\circ}$, and $60^{\circ}$. The thickness of the optical spacer layer is fixed as $\mathrm{x}=100 \mathrm{~nm}$. The calculated time-average Poynting

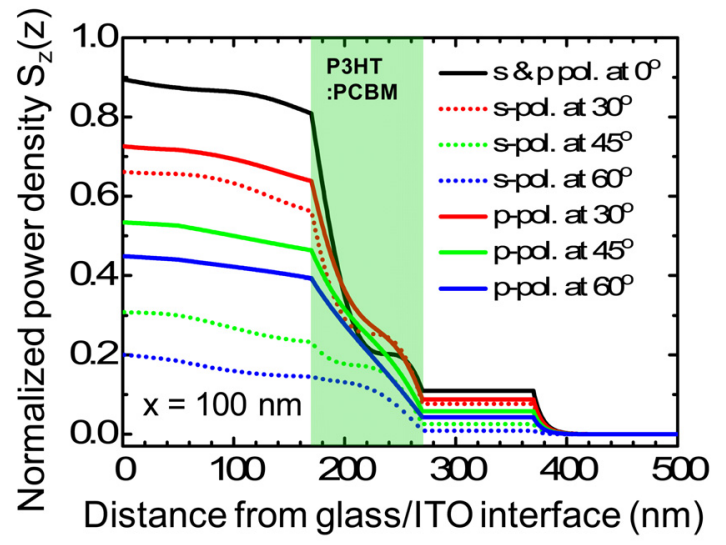

FIG. 4. Calculated time-average Poynting vectors for s- and p-polarized light at the incident angles of $0^{\circ}, 30^{\circ}, 45^{\circ}$, and $60^{\circ}$. The light green area indicates the P3HT:PCBM active region. The thickness of the optical spacer layer is fixed as $\mathrm{X}$ $=100 \mathrm{~nm}$. The calculated time-average Poynting vectors are normalized in reference to the incident optical power from the transparent glass.

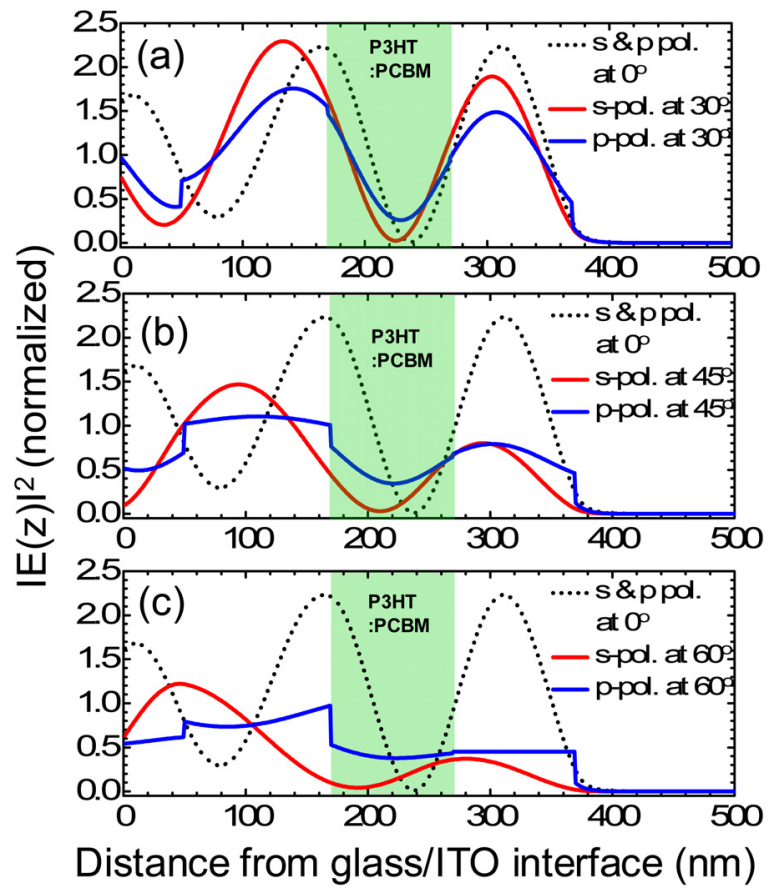

FIG. 5. Comparison of the calculated normalized electric field intensities between s- and p-polarized light at the incident angles of (a) $30^{\circ}$, (b) $45^{\circ}$, and (c) $60^{\circ}$. The light green area indicates the P3HT:PCBM active region. For reference, the electric field intensity distribution at normal incidence is shown in the black dotted line. The optical spacer layer is fixed as $\mathrm{x}=100 \mathrm{~nm}$. vectors are normalized in reference to the incident optical power from the transparent glass. As the incident angle increases, the value of $S_{z}(z)$ at the glass-ITO interface $(\mathrm{z}=0$ $\mathrm{nm})$ decreases because the front-reflection coefficient of the OSC multilayer, $\mathrm{r}_{0(M+1)}$ in Eq. (10a), becomes larger at the higher incident angle. The value of $S_{z}(z)$ with normal incidence $\left(\theta=0^{\circ}\right)$ is identical and degenerate for s- and p-polarized light. However, the value of $S_{z}(z)$ with oblique incidence of p-polarized light is larger than that with oblique incidence of s-polarized light. This higher transmittance of p-polarized light over s-polarized light at oblique incidence is similar to the transmission behavior at the boundary between two dielectric media, where the Brewster's angle (100\% transmission) is existent for p-polarized light [12].

Fig. 5 shows the comparison of the calculated normalized electric field intensities between s- and p-polarized light at the incident angles of (a) $30^{\circ}$, (b) $45^{\circ}$, and (c) $60^{\circ}$. For reference, the normalized electric field intensity distribution at normal incidence is shown in the black dotted line. The optical spacer layer thickness is $\mathrm{x}=100 \mathrm{~nm}$. The electric field intensity for p-polarized light $\left|E^{p}\right|^{2}=\left|E_{x}\right|^{2}+\left|E_{z}\right|^{2}$ is discontinuous at the interfaces because it has the electric field intensity component of $\left|E_{z}\right|^{2}$ normal to the interface. On the other hand, the electric field intensity for s-polarized light $\left|E^{s}\right|^{2}=\left|E_{y}\right|^{2}$ is continuous at the interfaces because it has only a transverse electric field intensity component. Fig. 6 shows the calculated optical power dissipations in the $\mathrm{z}$ direction for $\mathrm{s}-$ and $\mathrm{p}$-polarized light as a function of

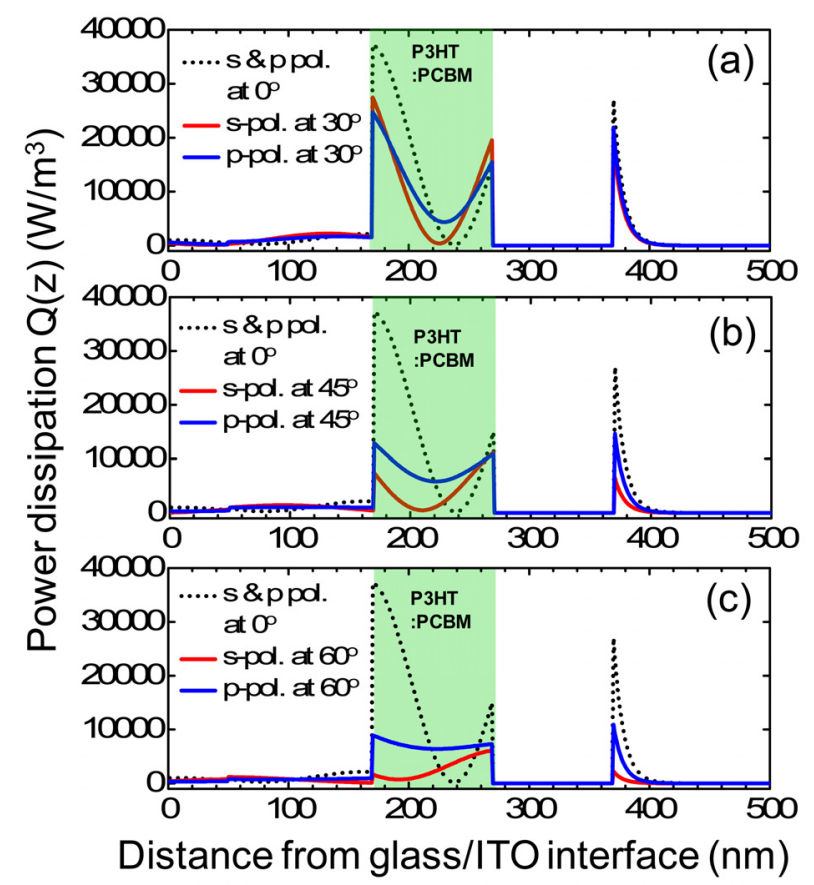

FIG. 6. Calculated optical power dissipations in the $\mathrm{z}$ direction for s- and p-polarized light as a function of the incident angle. The light green area indicates the P3HT:PCBM active region. The optical spacer layer is fixed as $\mathrm{x}=100 \mathrm{~nm}$. 
the incident angle. According to the definition of light absorptance in Eq. (27), p-polarized light shows the relatively higher light absorptance in the P3HT/PCBM active region than s-polarized light does This is more pronounced as the incidence angle increases from $30^{\circ}$ to $60^{\circ}$.

\section{CONCLUSION}

We presented comprehensive optical modeling and calculation results of thin-film OSC at oblique incidence of light. Based on the TMM, the simple expression for the optical power dissipation was derived at oblique incidence for s- and p-polarized light. Depending on light polarization, we calculated the spatial distribution of the electric field intensity, the timeaverage Poynting vector, and the optical power dissipation at various incidence angles. As the incidence angle increases, the optical power dissipation in the active region becomes smaller for both s- and p-polarized light. In the P3HT/PCBM active region, p-polarized light shows relatively higher light absorptance than s-polarized light does. This is more significant as the incidence angle increases up to $60^{\circ}$. We expect that our optical modeling results help to design more efficient OSC with light absorption efficiency improved, considering the optical interference effect of the OSC at oblique incidence.

\section{ACKNOWLEDGMENT}

This research was supported by the Kyung Hee University Research Fund in 2011 (KHU-20110921)

\section{REFERENCES}

1. H. Hoppe and N. S. Sariciftci, "Organic solar cells: an overview,” J. Mater Res. 19, 1924-1945 (2004).
2. V. Andersson, K. Tvingstedt, and O. Inganäs, "Optical modeling of a folded organic solar cell," J. Appl. Phys. 103, 094520 (2008).

3. J. Li, H. Yu, S. M. Wong, G. Zhang, X. Sun, P. G.-Q. Lo, and D.-L. Kwong, "Si nanopillar array optimization on $\mathrm{Si}$ thin films for solar energy harvesting," Appl. Phys. Lett. 95, 033102 (2009).

4. M. Niggemann, M. Riede, A. Gombert, and K. Leo, "Light trapping in organic solar cells," Phys. Stat. Sol. (a) 205, 2862-2874 (2008).

5. S. C. Kim and I. Sohn, "Simulation of energy conversion efficiency of a solar cell with gratings," J. Opt. Soc. Korea 14, 142-145 (2010).

6. L. A. A. Pettersson, L. S. Roman, and O. Inganäs, "Modeling photocurrent action spectra of photovoltaic devices based on organic thin films," J. Appl. Phys. 86, 487-496 (1999).

7. P. Peumans, A. Yakimov, and S. R. Forrest, "Small molecular weight organic thin-film photodetectors and solar cells," J. Appl. Phys. 93, 3693-3723 (2003).

8. N.-K. Persson, H. Arwin, and O. Inganäs, "Optical optimization of polyfluorene-fullerene blend photodiodes," J. Appl. Phys. 97, 034503 (2005).

9. G. Dennler, K. Forberich, M. C. Scharber, C. J. Brabec, I. Tomiš, K. Hingerl, and T. Fromherz, "Angle dependence of external and internal quantum efficiencies in bulk-heterojunction organic solar cells," J. Appl. Phys. 102, 054516 (2007).

10. D. Cheyns, B. P. Rand, B. Verreet, J. Genoe, J. Poortmans, and P. Heremans, "The angular response of ultrathin film organic solar cells,” Appl. Phys. Lett. 92, 243310 (2008).

11. A. Meyer and H. Ade, "The effect of angle of incidence on the optical field distribution within thin film organic solar cells," J. Appl. Phys. 106, 113101 (2009).

12. B. E. A. Saleh and M. C. Teich, Fundamentals of Photonics, 2nd ed. (Wiley, New Jersey, USA, 2007), Chapter 6.

13. M. Agrawal and P. Peumans, "Broadband optical absorption enhancement through coherent light trapping in thin-film photovoltaic cells," Opt. Express 16, 5385 (2008).

14. P. D. Andersen, J. C. Skårhøj, J. W. Andreasen, and F. C. Krebs, "Investigation of optical spacer layers from solution based precursors for polymer solar cells using x-ray reflectometry," Opt. Mater. 31, 1007-1012 (2009). 